\title{
Consumo de drogas en adolescentes de la Comunidad de Madrid
}

\author{
Muñoz Rivas, M.J.*; Cruzado RodríGuez, J.A.**; Graña Gómez, J.L.*** \\ * Profesora asociada de la Universidad Complutense de Madrid y de la Universidad San Pablo-CEU de Madrid. \\ * Profesor titular de Psicología de la Salud y Director del Departamento de Psicología Clínica de la Universidad Complutense de Madrid. \\ ** Profesor titular de Psicopatología de la Universidad Complutense de Madrid.
}

\section{Resumen}

En el presente estudio se analiza la prevalencia de consumo de distintas drogas de comercio ilegal (cannabis, cocaína, drogas sintéticas y médicas) en adolescentes de la Comunidad Autónoma de Madrid. Para ello, se ha utilizado una muestra de 1.570 sujetos de ambos sexos (54,4\% hombres y $45,6 \%$ mujeres) con edades comprendidas entre los 14 y los 18 años. Los resultados muestran que el cannabis es la sustancia más consumida por los jóvenes ofreciendo una prevalencia de consumo muy superior a la obtenida por el resto de las sustancias estimadas. El consumo de este tipo de drogas mantiene una relación directa con la edad, siendo el grupo de jóvenes de entre 16 y 18 años, el de mayor riesgo en comparación con el de menor edad.

Palabras Clave: adolescentes, consumo, drogas, ilegales, prevalencia.

\section{Summary}

In this study the focus of analysis is the prevalence of consumption of ilegal drugs (cannabis, cocaine, synthetic and medical drugs) in adolescents of Madrid. A sample of 1570 subjectos of boths sexes was studied $(54.4 \%$ were boys and $45.6 \%$ were girls) within a range from 14 to 18 years of age. The results pointed out that cannabis was the drug most consumed by young people showing a prevalence of consumption higher than that obtained by the other ilegal drugs. The use of this type of drugs keeps a direct relationship with age, the group of 16 to 18 years old being the one of higher risk comparing with those of lower age.

Key words: Adolescents, consumption, drugs, ilegal, prevalence Adolescents, consumption, drugs, ilegal, prevalence

\section{INTRODUCCIÓN}

$\mathbf{L}$ a situación de las drogodependencias en nuestro país ha experimentado, en los últimos años, cambios importantes no sólo en cuanto a los porcentajes globales de consumidores en la población en general sino también en el tipo de sustancia consumida (PNSD, 1995). Tal es el caso de la heroína, que ha pasado de ser una de las sustancias más consumidas a figurar entre las que ofrecen menores porcentajes, siendo desplazada por otras más novedosas como son las drogas de síntesis (PNSD, 1995; PNSD, 1996). En este sentido, a modo de ejemplo, se observa que los consumidores actuales no sólo lo son de sustancias como el éxtasis o las pastillas (se conoce que en 1995, la proporción de españoles mayores de 14 años que las había consumido durante el ultimo año superaba ya el $1 \%)$, sino que, además, incluyen en sus pautas de consumo otras sustancias como el alcohol, el cannabis, la cocaína y/o los alucinógenos (PNSD, 1996). Se trata, pues, de una situación compleja en la que una gran proporción de la población consumidora abusa no sólo de una sino de varias sustancias al mismo tiempo, lo que agrava tanto su rehabilitación como, lo que es más importante, la prevención del consumo en todos sus niveles (individual, familiar, comunitario).

Es ésta una situación realmente alarmante si tenemos en cuenta que muchos de estos aspectos son también relevantes para la población adolescente, puesto que comparte con la adulta el consumo abusivo de sustancias psicoactivas. Es necesario considerar que, en estas edades, no sólo se establecen los primeros contactos con las sustancias adictivas, sino la instauración y consolidación de patrones estables que pueden marcar el paso hacia el consumo de otras drogas e, incluso, la aparición temprana de problemas relacionados con la salud o el ajuste social, que llegan a afectar de forma determinante en la edad adulta.

Centrándonos en el consumo de drogas de comercio ilegal, en la última década, el análisis de varios estudios realizados en el ámbito nacional (ECERS, 1990; 
INJUVE, 1993; PNSD, 1994; PNSD, 1996) y otros específicos de la Comunidad Autónoma de Madrid (CAM, 1996, 1997), informan que, después del alcohol y el tabaco, el cannabis es la droga más extendida entre los adolescentes y la que ha experimentado un mayor incremento entre los jóvenes de nuestro país (Tabla 1). En pocos años esta sustancia ha pasado de ser minoritariamente consumida (en 1990, sólo el $4 \%$ de los encuestados afirmaban haberla consumido en el último mes) a ser una de las más habituales y más conocidas por los escolares (en 1996, el 16\% de los entrevistados la consume mensualmente). Tal y como advierte el Plan Nacional sobre Drogas (1994), de todas las drogas ilegales, esta sustancia es la que posee mayores prevalencias de consumo, confirmándose como la de mayor penetración en la población más joven.

Tabla 1. Proporción de consumidores de cannabis en la población adolescente (1990-1996).

\begin{tabular}{|c|c|c|c|c|c|}
\hline Año & Encuesta & Edad & Muestra & $\%$ Consumidores & Rango edad/Edad de inicio \\
\hline 1990 & ECERS & 11-18 años & 4.393 & $\begin{array}{r}4.0 \%{ }^{\mathrm{A}} \\
12.0 \%{ }^{\mathrm{B}} \\
17.0 \%^{\mathrm{C}}\end{array}$ & $\begin{array}{l}13 \text { / } 12.7 \text { años } \\
15 \text { / } 14.4 \text { años } \\
17 \text { / } 15.8 \text { años }\end{array}$ \\
\hline 1993 & INJUVE & 15-29 años & 1.201 & $\begin{array}{r}29.5 \%^{C} \\
7.2 \%{ }^{D}\end{array}$ & $\begin{array}{l}\text { 15-16 / } 15.2 \text { años } \\
\text { 17-19 / } 16.5 \text { años } \\
\text { 20-24 / } 17.2 \text { años } \\
\text { 25-29 / } 18.0 \text { años }\end{array}$ \\
\hline 1994 & PNSD & 14-18 años & 21.094 & $\begin{array}{l}12.2 \%^{\mathrm{A}} \\
18.1 \%^{\mathrm{B}} \\
20.8 \%^{\mathrm{C}}\end{array}$ & $\begin{array}{r}14-15 \text { / } 14.0 \text { años } \\
16 \text { / } 14.9 \text { años } \\
17-18 \text { / } 15.8 \text { años } \\
14-18 \text { / } 15.1 \text { años }\end{array}$ \\
\hline 1996 & PNSD & 14-18 años & 19.191 & $\begin{array}{l}15.7 \%^{\mathrm{A}} \\
23.2 \%^{\mathrm{B}} \\
26.0 \%^{\mathrm{C}}\end{array}$ & 1418 / 15.0 años \\
\hline 1996 & CAM & 15-16 años & 1.799 & $22.9 \%^{B}$ & - \\
\hline 1997 & CAM & 15-16 años & 2.602 & $24.0 \%{ }^{B}$ & - \\
\hline
\end{tabular}

A. Ha consumido cannabis durante el último mes

B. Ha consumido cannabis durante el último año

C. Ha consumido cannabis alguna vez en su vida

D. Usuarios habituales: relación con la sustancia más o menos frecuente pero permanente y activa

Así pues, si se analizan los datos recogidos por los dos estudios realizados por el PNSD en 1994 y 1996, en este último año reconocían haber consumido esta sustancia en los últimos 12 meses un $5 \%$ más de jóvenes que en 1994. Estos datos adquieren mayor relevancia si se tiene en cuenta que el cannabis suele ser la sustancia que da acceso al consumo de otras de mayor poder adictivo que tienen consecuencias más nocivas para los consumidores como son la cocaína o las drogas de síntesis. Además, la edad de inicio del consumo de esta sustancia suele ser mayor que en el caso del alcohol y del tabaco, lo que nos advierte, probablemente, de la importancia de la disponibilidad de las sustancias de consumo y del fenómeno de escalada dentro del consumo de drogas. Con respecto a esta variable, los estudios señalan (al igual que ocurre en el caso del alcohol y el tabaco) que la proporción de con- sumidores de cannabis también parece aumentar de forma notable en función de la edad de los mismos (en 1996, la proporción de jóvenes que habían consumido esta sustancia el año previo a la encuesta pasó del 8.3\% a los 14 años al 40.3\% a los 18 años), observándose un incremento mayoritario a los 16 años y es, a partir de los 18, cuando se encuentra un mayor número de jóvenes consumidores habituales.

Asimismo, considerando el consumo de psicoestimulantes, se observa cómo, en pocos años, el consumo de sustancias como la cocaína, los alucinógenos o el éxtasis ha pasado de ser casi inexistente a ocupar un puesto importante en las listas de las drogas ilegales más utilizadas por los jóvenes españoles (Tabla 2). Así, por ejemplo, el porcentaje de consumidores de alucinógenos en el año previo a la encuesta pasó del $0.1 \%$ en 1990 al 2.6\% en 1996 y, lo que es más importante, 
sustancias como las drogas sintéticas, que prácticamente no se conocían a comienzos de la década, son ahora consumidas de forma habitual por el $2.2 \%$ de la población de jóvenes de 14 a 18 años (PNSD, 1996). En este sentido, en los últimos años, es posible hablar de un incremento significativo del consumo de todas las sustancias analizadas, especialmente en el caso de los alucinógenos y los derivados anfetamínicos incluido el éxtasis (en 1996, el 3.9\% de los encuestados lo había consumido durante el último año).

\section{Tabla 2. Proporción de consumidores de psicoestimulantes en la población adolescente}

(1990-1996).

\begin{tabular}{|c|c|c|c|c|c|c|c|c|c|}
\hline Año & Encuesta & Edad & Muestra & ranquil. & Anfetam. & Cocaína & D. Sintéticas & Alucinog. & Heroína \\
\hline 1990 & ECERS & 11-18 años & 4.393 & $\begin{array}{l}0.2 \%^{A} \\
0.8 \%^{\mathrm{C}}\end{array}$ & $\begin{array}{l}0.3 \%^{A} \\
1.1 \%^{\mathrm{C}}\end{array}$ & $\begin{array}{l}0.4 \%^{A} \\
0.9 \%^{\mathrm{C}}\end{array}$ & - & $\begin{array}{l}0.1 \%^{A} \\
0.3 \%^{C}\end{array}$ & $\begin{array}{l}0.1 \%^{\mathrm{A}} \\
0.4 \%^{\mathrm{C}}\end{array}$ \\
\hline 1993 & INJUVE & 15-29 años & 1.201 & - & $\begin{array}{l}4.8 \%^{\mathrm{C}} \\
1.3 \%^{\mathrm{D}}\end{array}$ & $\begin{array}{c}7.8 \%^{\mathrm{c}} \\
2.0 \%^{\mathrm{D}}\end{array}$ & $\begin{array}{l}4.3 \%^{\mathrm{c}} \\
1.1 \%^{\mathrm{D}}\end{array}$ & 二 & $\begin{array}{l}1.6 \%^{\mathrm{C}} \\
0.4 \%^{\mathrm{D}}\end{array}$ \\
\hline 1994 & PNSD & 14-18 años & 21.094 & $\begin{array}{l}2.5 \%^{\mathrm{A}} \\
4.3 \%^{\mathrm{B}} \\
5.9 \%^{\mathrm{C}}\end{array}$ & $\begin{array}{l}2.1 \%^{\mathrm{A}} \\
3.3 \%^{\mathrm{B}} \\
4.1 \%^{\mathrm{C}}\end{array}$ & $\begin{array}{l}1.0 \%^{\mathrm{A}} \\
1.7 \%^{\mathrm{B}} \\
2.4 \%^{\mathrm{C}}\end{array}$ & $\begin{array}{l}2.0 \%^{\mathrm{A}} \\
3.0 \%^{\mathrm{B}} \\
3.5 \%^{\mathrm{C}}\end{array}$ & $\begin{array}{l}2.4 \%^{\mathrm{A}} \\
4.0 \%^{\mathrm{B}} \\
4.7 \%^{\mathrm{C}}\end{array}$ & $\begin{array}{l}0.2 \%^{\mathrm{A}} \\
0.3 \%^{\mathrm{B}} \\
0.5 \%^{\mathrm{C}}\end{array}$ \\
\hline 1996 & PNSD & 14-18 años & 19.191 & $\begin{array}{l}2.2 \%^{A} \\
4.4 \%^{B} \\
5.9 \%^{C}\end{array}$ & $\begin{array}{l}2.4 \%^{A} \\
4.1 \%^{B} \\
4.9 \%^{C}\end{array}$ & $\begin{array}{l}1.5 \%^{A} \\
2.6 \%^{B} \\
3.2 \%^{C}\end{array}$ & $\begin{array}{l}2.2 \%^{A} \\
3.9 \%^{B} \\
5.1 \%^{C}\end{array}$ & $\begin{array}{l}2.6 \%^{A} \\
5.3 \%^{B} \\
6.5 \%^{C}\end{array}$ & $\begin{array}{l}0.6 \%^{\mathrm{A}} \\
0.4 \%^{\mathrm{B}} \\
0.3 \%^{\mathrm{C}}\end{array}$ \\
\hline 1996 & CAM & 15-16 años & 1.799 & $3.9 \%^{\mathrm{B}}$ & $2.4 \%^{B}$ & $2.7 \%^{\mathrm{B}}$ & $3.2 \%^{B}$ & $5.5 \%^{\mathrm{B}}$ & $0.5 \%^{\mathrm{B}}$ \\
\hline 1997 & CAM & 15-16 años & 2.602 & $2.6 \%^{\mathrm{B}}$ & $1.7 \%^{\mathrm{B}}$ & $2.3 \%^{B}$ & $2.7 \%^{\mathrm{B}}$ & $4.4 \%^{B}$ & $0.6 \%^{\mathrm{B}}$ \\
\hline
\end{tabular}

A. Ha consumido la sustancia en el último mes

B. Ha consumido la sustancia en el último año

C. Ha consumido la sustancia alguna vez en la vida

D. Usuarios habituales: relación con la sustancia más o menos frecuente pero permanente y activa

Es especialmente relevante señalar la tendencia contraria observada para el consumo de heroína, ya que los datos aportados señalan que, atendiendo a los porcentajes de consumidores que se inician en su consumo en la etapa adolescente y juvenil, el uso de esta sustancia parece haberse estabilizado e incluso decrecer en comparación con otro tipo de drogas (en 1996, en la Comunidad de Madrid, el porcentaje de consumidores anuales de heroína se situaba en torno al $0.5 \%$ de los entrevistados frente al $5.5 \%$ de consumidores de alucinógenos).

Con respecto a la edad de inicio, se observa nuevamente que los jóvenes comienzan a experimentar y a consumir drogas ilegales a edades más avanzadas (en torno a los 15.5 años), pero es necesario atender a los datos ofrecidos por el PNSD en 1996 en comparación con los obtenidos en 1994, que parecen indicar una preocupante disminución de la edad de inicio en el uso de drogas de síntesis y tranquilizantes, situándose en los 14.9 y los 13.8 años, respectivamente. En lo que respecta a la prevalencia del consumo en función de la edad de los encuestados, se constata que la proporción de los consumidores de la mayor parte de las drogas ilegales aumenta de forma progresiva entre los 14 y los 18 años. Así, por ejemplo, en sustancias como la cocaína este incremento se acentúa a los 16 años, llegando a ser más elevado en el paso de los 17 a los 18 años, sobre todo cuando hablamos de un consumo no habitual (en 1994, pasó de un $0.4 \%$ de consumidores a los 14 años al $5.0 \%$ de los encuestados con 18 años). Esta misma tendencia se observa también en otras drogas como el éxtasis (en 1996, pasó de ser consumida por el $1 \%$ de los jóvenes con 14 años a consumirla el $8.2 \%$ de los encuestados con 18 años) y los alucinógenos, que son utilizados de forma mayoritaria entre los 17 y los 18 años.Con respecto a la posible influencia de la variable sexo, se observa que la proporción de consumidores de la mayoría de las drogas consideradas es más alta en varones que en mujeres, reduciéndose las diferencias cuando se estima el consumo más reciente. Es importante señalar que, en el caso de los tranquilizantes, la tendencia observada es contraria a la obtenida para el resto de las sustancias siendo las mujeres las usuarias más importantes (en 1996, el 5.6\% de la entrevistadas consumió algún tipo de tranquilizantes o 
pastillas para dormir sin prescripción médica, mientras que sólo el $3.1 \%$ de los chicos había realizado cualquiera de estos consumos).

Teniendo como punto de partida las consideraciones señaladas, el estudio de investigación que aquí se presenta se centra en determinar la prevalencia de consumo de determinadas sustancias de comercio ilegal en la población adolescente de la Comunidad de Madrid, mostrando el tipo de consumo realizado y las sustancias de mayor utilización, con el objetivo de especificar la situación actual y facilitar, en la medida de lo posible, la adecuación de los esfuerzos preventivos realizados en este área.

\section{MATERIAL Y MÉTODOS}

El punto de partida del presente estudio fue la selección de los centros escolares donde se llevó a cabo la investigación. Para ello, se establecieron una serie de criterios de inclusión/exclusión mínimos que debían cumplir los centros de enseñanza participantes. En este primer paso, para ser considerados, todos los centros debían cumplir los siguientes requisitos: (1) impartir todos los cursos de Enseñanza Secundaria o primero y segundo de Formación Profesional para poder acceder a la población de referencia; (2) tener un número de alumnos no inferior a mil. Con ello, se eliminaban del espectro posible todos aquellos centros cuya muestra no fuera representativa de la población de estudio bien por no contar con todos los cursos de Educación Secundaria o Formación Profesional, o bien por no tener el suficiente número de alumnos en cada una de las clases, lo que, de nuevo, afectaría a la representatividad de la muestra seleccionada; (3) estar ubicados en distintas zonas de la Comunidad de Madrid. El objetivo era obtener una muestra en la que estuvieran representados, en la medida de lo posible, todos los niveles socioeconómicos y culturales; (4) disponer de un Departamento de Orientación a través del cual poder organizar la implementación del proceso de evaluación y, (5) mostrar un interés inicial en el desarrollo de estudios relacionados con la problemática juvenil con respecto al uso de drogas en un primer contacto telefónico en el que se exponían las bases de la investigación, facilitando, así, la posibilidad de desarrollarla dentro del ámbito escolar.

Una vez realizado este primer paso, se dispuso de un total de diez institutos que cumplían los requisitos especificados y tenían características similares y, por lo tanto, eran posibles candidatos a participar en el estudio. De todos los centros preseleccionados, se escogieron al azar los seis que, finalmente, colaboraron en la investigación y de los cuales se extrajo la muestra de sujetos analizada: (1) Instituto Nacional de Bachillerato Virgen de la Paloma (19,7\%); (2) Instituto
Nacional de Bachillerato Ramiro de Maeztu (10,8\%); (3) Colegio Franciscanas de Montpellier (7,4\%); (4) Instituto Nacional de Bachillerato Jorge Guillén (Alcorcón) (15,8\%); (5) Instituto Nacional de Bachillerato Majadahonda III (Majadahonda) (28,3\%) y, (6) Instituto Nacional de Educación Secundaria "La Arboleda" (Alcorcón) $(17,9 \%)$.

Dentro de cada uno de los centros de enseñanza, el muestreo se realizó por conglomerados tomando el aula como unidad muestral, eligiéndose al azar las aulas participantes de cada curso de Educación Secundaria y Formación Profesional. La aplicación del instrumento de evaluación se realizó siempre de forma colectiva contando con aquellos alumnos que estuvieran presentes en ese momento, ya que se pidió a los tutores que no avisaran al grupo hasta el mismo día de la aplicación. En cada aula estaban siempre presentes dos de los evaluadores entrenados en ausencia de los profesores para evitar que el alumnado se sintiera influido de alguna manera, por su presencia a la hora de contestar a ciertas preguntas relacionadas con el consumo o con el ambiente escolar. La duración de la prueba era aproximadamente de 50 minutos, pero se dispuso, en los casos en que fue necesario, de los 10 minutos disponibles para el descanso entre clases.

Una vez llevado a cabo este primer paso, se obtuvo una muestra de 1.742 adolescentes que participaron de forma voluntaria en el desarrollo de la prueba y eran conocedores de que ésta formaba parte de una investigación realizada desde la Facultad de Psicología de la Universidad Complutense de Madrid.

\section{Instrumentos}

Teniendo en cuenta los criterios generales de la investigación se creó un instrumento de evaluación que se adecuase a los objetivos perseguidos. Así pues, el cuestionario resultante incluyó una primera parte presentada en forma de encuesta que recogía los datos relativos al consumo de sustancias (57 ítems) y, una segunda parte, en la que se presentaban un total de cinco escalas de evaluación psicológica. En el primer apartado de análisis de las variables relacionadas con el uso y abuso de sustancias se consideró el consumo de distintas drogas a través de diversos parámetros temporales como el uso de cada una de ellas alguna vez en la vida, en los últimos seis meses o en los últimos treinta días, donde el sujeto debía contestar afirmativa o negativamente $y$, en el caso de utilizarlas frecuentemente, indicar la frecuencia con que lo hacía. Además, se registró la edad en la que comenzó el consumo en el caso de haber probado cualquiera de las sustancias indicadas.

Se incluyó el análisis de sustancias de comercio legal (alcohol y tabaco) e ilegal, objeto del presente 
estudio. Entre ellas, además del cannabis, la cocaína y las drogas de síntesis, se decidió incorporar un grupo de drogas denominadas médicas conformado por las anfetaminas y derivados de uso comercial (además de las anfetaminas se consideró el uso de otro tipo de medicamentos comunes que pudieran provocar el mismo efecto estimulante como son determinados compuestos de fácil acceso para el tratamiento del insomnio o para el adelgazamiento, por ser más utilizadas por padres y adultos y poderse adquirir a un bajo precio no porque no requieran prescripción médica para su obtención), los fármacos antirreumáticos (que podían también ser de los más utilizados por la población de jóvenes adolescentes al ser indicados para el tratamiento de dolencias típicas de esta edad provocados por el deporte o la actividad física), los tranquilizantes y, finalemente, los derivados morfínicos (además de la heroína se consideraron también el uso de otra serie de fármacos con una composición derivada de la codeína o la morfina de posible uso común por su poder analgésico).

\section{RESULTADOS}

Una vez realizadas las encuestas de forma voluntaria por el total de los adolescentes participantes en la investigación (1.742), se eliminaron un total de 172 encuestas por no haber sido contestados correctamente cada uno de los ítems o por carecer de alguno de los datos necesarios para su codificación. Así pues, se trabajó con una muestra final de 1.570 sujetos de ambos sexos ( $54.4 \%$ hombres y $45.6 \%$ mujeres) con edades comprendidas entre los 14 y los 18 años (edad media de 15.83 años).

Tomando como referencia el grupo de consumidores en los últimos treinta días de cualquiera de las sustancias de comercio ilegal estimadas, el estudio detallado de las proporciones de consumidores y su relación con el sexo y la edad de los mismos se presenta en la tabla 3. De forma genérica, se observa nuevamente como el cannabis, los estimulantes, los derivados morfínicos y las drogas de síntesis son las sustancias más consumidas por los jóvenes de 14 a 18 años.

Tabla 3. Proporción de jóvenes con consumo ilegal en los últimos 30 días según edad y sexo.

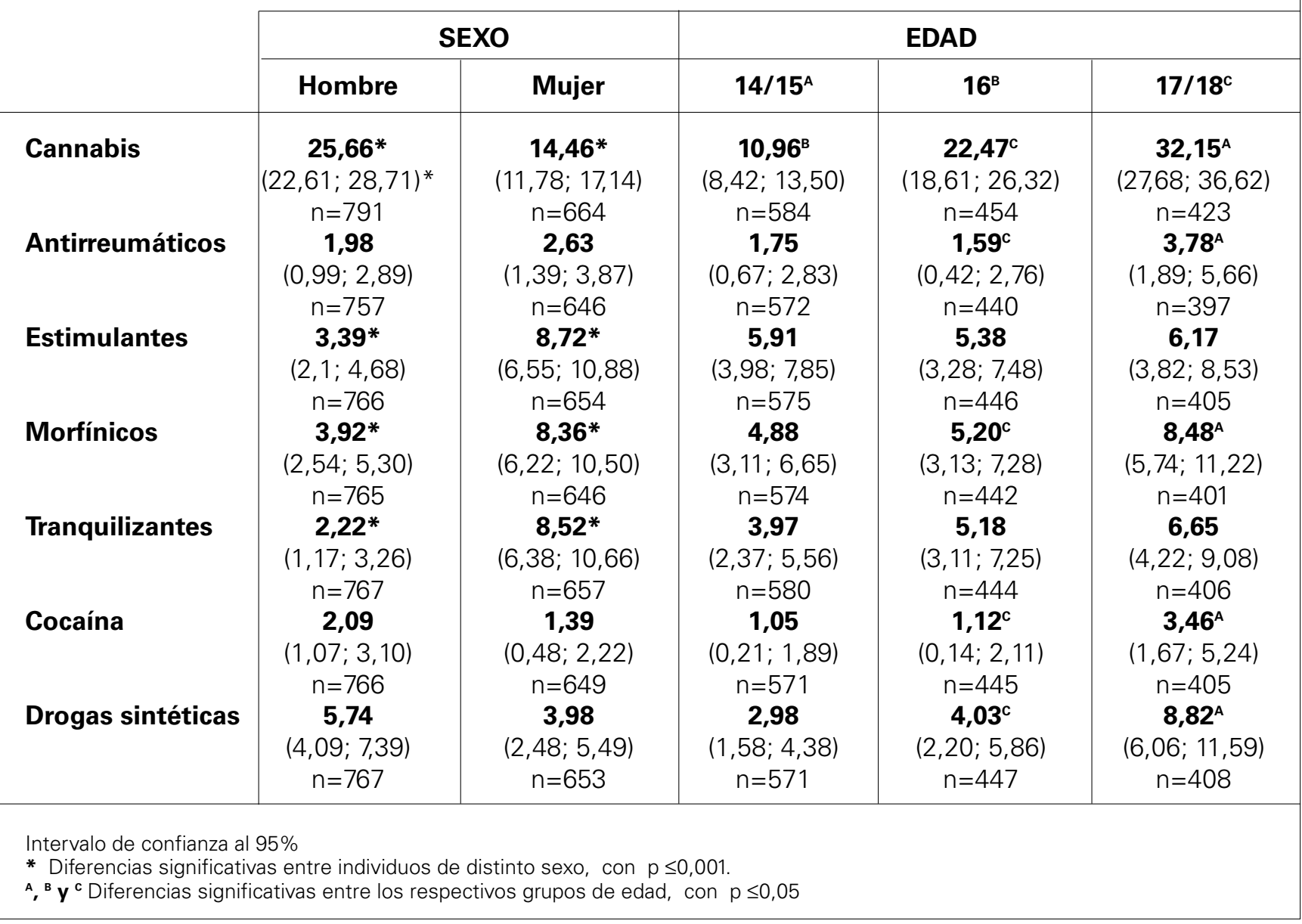

Atendiendo al análisis de las relaciones entre el sexo de los encuestados y el consumo de cada una de las sustancias, los resultados son estadísticamente significativos y superiores en el caso de los varones sólo para el consumo de cannabis [25.66 vs. 14.46, $\left.\mathrm{X}^{2}=34.69, \mathrm{p} \leq 0.001\right]$ mientras que son las mujeres las que muestran mayores porcentajes de consumo en sustancias como los estimulantes [3.39 vs. 8.72, 
$X^{2}=23.25, p \leq 0.001$ ], los derivados morfínicos [3.92 vs. 8.36, $\left.X^{2}=14.95, p \leq 0.001\right]$ y los tranquilizantes [2.22 vs. 8.52, $\left.X^{2}=30.89, p \leq 0.001\right]$. En el caso de la cocaína y las drogas de síntesis, a pesar de que hay una mayor proporción de varones consumidores, no se establecen diferencias significativas con respecto al grupo de mujeres, al igual que ocurre en el caso de los fármacos antirreumáticos en el que son las mujeres las que los consumen en mayor medida aunque este incremento porcentual no sea significativo en comparación con el grupo de varones.

Asimismo, la edad también tiene una relación estadísticamente significativa con la prevalencia de consumo de cualquiera de las sustancias estimadas ya que la proporción de consumidores es mayor cuanto mayor es la edad de los encuestados, llegando incluso, como ocurre con el cannabis, la cocaína y las drogas de síntesis, a ser tres veces superior a los 17/18 años si se compara con la del grupo de menor edad. De cualquier forma, es necesario señalar las altas prevalencias de consumo obtenidas puesto que más del $10 \%$ de los adolescentes de 14 a 15 años en el caso del cannabis y casi el $6 \%$ en el de los estimulantes, ha consumido la sustancia en el mes previo a la realización de la encuesta.

Los resultados obtenidos en el estudio de la distribución del consumo según el tipo de sustancia de comercio ilegal y la frecuencia de uso en los consumidores en el último mes se presentan en la tabla 4.

\begin{tabular}{|c|c|c|c|c|c|c|c|}
\hline & $\begin{array}{c}\text { Cannabis } \\
(n=302)\end{array}$ & $\begin{array}{l}\text { Antirreumát. } \\
\qquad(\mathrm{n}=32)\end{array}$ & $\begin{array}{l}\text { Estimulantes } \\
\qquad(n=83)\end{array}$ & $\begin{array}{c}\text { Morfínicos } \\
\quad(n=85)\end{array}$ & $\begin{array}{c}\text { Tranquil. } \\
(n=73)\end{array}$ & $\begin{array}{c}\text { Cocaína } \\
(n=25)\end{array}$ & $\begin{array}{l}\text { D.sintéticas } \\
\quad(n=71)\end{array}$ \\
\hline$<1 \mathrm{vez} /$ semana & $\begin{array}{c}\mathbf{4 8 , 0} \% \\
(n=145)\end{array}$ & $\begin{array}{l}\mathbf{4 3 , 8} \% \\
(n=14)\end{array}$ & $\begin{array}{l}33,7 \% \\
(n=28)\end{array}$ & $\begin{array}{l}\mathbf{6 3}, 5 \% \\
(n=54)\end{array}$ & $\begin{array}{l}\mathbf{6 5 , 8} \% \\
(n=48)\end{array}$ & $\begin{array}{l}\mathbf{6 4 , 0} \% \\
(n=16)\end{array}$ & $\begin{array}{l}73,2 \% \\
(n=52)\end{array}$ \\
\hline $1 \mathrm{vez} / \mathrm{semana}$ & $\begin{array}{l}\mathbf{2 0}, \mathbf{5} \% \\
(n=62)\end{array}$ & $\begin{array}{c}15,6 \% \\
(n=5)\end{array}$ & $\begin{array}{l}15,7 \% \\
(n=13)\end{array}$ & $\begin{array}{l}\mathbf{9 , 4 \%} \\
(n=8)\end{array}$ & $\begin{array}{l}\mathbf{8}, 2 \% \\
(n=6)\end{array}$ & $\begin{array}{c}12,0 \% \\
(n=3)\end{array}$ & $\begin{array}{l}\mathbf{1 8}, \mathbf{3} \% \\
(n=13)\end{array}$ \\
\hline $2 / 6$ veces/semana & $\begin{array}{l}17,9 \% \\
(n=54)\end{array}$ & $\begin{array}{c}15,6 \% \\
(n=5)\end{array}$ & $\begin{array}{c}10,8 \% \\
(n=9)\end{array}$ & $\begin{array}{l}3,5 \% \\
(n=3)\end{array}$ & $\begin{array}{l}\mathbf{8 , 2} \% \\
(n=6)\end{array}$ & $\begin{array}{l}12,0 \% \\
(n=3)\end{array}$ & $\begin{array}{l}\mathbf{4 , 2} \% \\
(n=3)\end{array}$ \\
\hline 1 vez diaria & $\begin{array}{c}7,3 \% \\
(n=22)\end{array}$ & $\begin{array}{c}12,5 \% \\
(n=4)\end{array}$ & $\begin{array}{l}\mathbf{1 4 , 5} \% \\
(n=12)\end{array}$ & $\begin{array}{l}8,2 \% \\
(n=7)\end{array}$ & $\begin{array}{l}\mathbf{8}, 2 \% \\
(n=6)\end{array}$ & $\begin{array}{l}\mathbf{4 , 0} \% \\
(n=1)\end{array}$ & $\begin{array}{l}\mathbf{0 , 0 \%} \\
(n=0)\end{array}$ \\
\hline$>1$ vez diaria & $\begin{array}{c}\mathbf{6 , 3} \% \\
(n=19)\end{array}$ & $\begin{array}{c}12,5 \% \\
(n=4)\end{array}$ & $\begin{array}{l}25,3 \% \\
(n=21)\end{array}$ & $\begin{array}{l}15,3 \% \\
(n=13)\end{array}$ & $\begin{array}{l}9,6 \% \\
(n=7)\end{array}$ & $\begin{array}{l}\mathbf{8 , 0} \% \\
(n=2)\end{array}$ & $\begin{array}{l}4,2 \% \\
(n=3)\end{array}$ \\
\hline
\end{tabular}

Como puede observarse, dentro del grupo de consumo reciente (en el último mes), la sustancia más consumida por los jóvenes son los estimulantes puesto que un $66.3 \%$ de los que los consumen lo hacen con una frecuencia al menos semanal (es decir, consumen esta sustancia diariamente o de 1 a 6 veces por semana), el cannabis cuyo porcentaje de consumidores semanales supone el $62.0 \%$ de la población de referencia y los fármacos antirreumáticos que supera el $56 \%$. De forma contraria, en el resto de las sustancias, el porcentaje de jóvenes que consumen con una frecuencia superior a la semanal supera a la proporción de consumidores semanales especialmente en el consumo de drogas de síntesis (un $73.2 \%$ frente al $26.8 \%)$.

Además de los datos comentados, se analizó la edad media de inicio en el consumo de cada una de las sustancias estimadas junto con el patrón de continuidad en el mismo según el sexo y la edad de los consumidores.

Así pues, en el consumo de cannabis, la edad media de inicio se sitúa en los 14.9 años, sin que exis- tan diferencias significativas entre hombres y mujeres $[14.95$ vs. $14.89, t(501)=0.53$, n.s. ], señalando una edad de comienzo equivalente en ambos casos (Tabla 5).

Con respecto a la edad, se detecta un incremento significativo de la edad de comienzo en el uso de cannabis en los grupos de 16 a 18 años con respecto al de menor edad (en el grupo de 17/18 años, la edad media de inicio supera casi en dos años a la obtenida para el grupo de consumidores de 14/15 años).

En esta misma sustancia, el $75.7 \%$ de los que han probado el cannabis alguna vez en la vida lo ha seguido haciendo en los últimos seis meses y, de este porcentaje, el $80.5 \%$ siguen consumiendo en el último mes. En este caso, los hombres [88.26 vs. $68.08, Z=$ $4.80, p \leq 0.05$ ] y el grupo de mayor edad (17/18 años), introducen diferencias significativas con respecto al resto de los grupos ya que son las categorías en las que la continuidad es mayor.

En el consumo de fármacos antirreumáticos (Tabla 6), la edad media de inicio se sitúa en los 14.1 años, sin que existan diferencias significativas entre hombres y mujeres [14.58 vs. $13.69, t(50)=1.67$, n.s.]. 
Tabla 5. Edad media de inicio y continuidad en el consumo de cannabis según sexo y edad.

\begin{tabular}{|c|c|c|c|c|c|c|}
\hline & \multicolumn{6}{|c|}{ CANNABIS } \\
\hline & \multicolumn{3}{|c|}{ SEXO } & \multicolumn{3}{|c|}{ EDAD } \\
\hline & Total & Hombres & Mujeres & $14 / 15^{A}$ & $16^{\mathrm{B}}$ & $17 / 18^{c}$ \\
\hline Edad media de inicio & $\begin{array}{c}\mathbf{1 4 , 9 4} \\
(d t=1.29) \\
n=506\end{array}$ & $\begin{array}{c}\mathbf{1 4 , 9 5} \\
(\mathrm{dt}=1.31) \\
n=303\end{array}$ & $\begin{array}{c}\mathbf{1 4 , 8 9} \\
(d t=1.25) \\
n=200\end{array}$ & $\begin{array}{c}\mathbf{1 4 , 0 4} \\
(\mathrm{dt}=0.92) \\
\mathrm{n}=117\end{array}$ & $\begin{array}{c}\mathbf{1 4 , 8 3}^{\mathrm{A}} \\
(\mathrm{dt}=1.04) \\
n=177\end{array}$ & $\begin{array}{c}\mathbf{1 5}, \mathbf{5} 2^{\mathrm{A}, \boldsymbol{B}} \\
(\mathrm{dt}=1.34) \\
n=212\end{array}$ \\
\hline $\begin{array}{l}\text { Continuidad consumo } \\
\text { (Consumo reciente/consumo } \\
\text { alguna vez)*100 } \\
\text { (Consumo frecuente/consumo } \\
\text { reciente)*100 }\end{array}$ & $\begin{array}{l}75,71 \\
80,59\end{array}$ & $\begin{array}{c}76,92 \\
88,26 *\end{array}$ & $\begin{array}{c}73,82 \\
68,08 *\end{array}$ & $\begin{array}{l}78,76 \\
71,91\end{array}$ & $\begin{array}{l}76,74 \\
77,27\end{array}$ & $\begin{array}{c}73,20 \\
88,88^{A, B}\end{array}$ \\
\hline
\end{tabular}

Tabla 6. Edad media de inicio y continuidad en el consumo de fármacos antirreumáticos según sexo y edad.

\begin{tabular}{|c|c|c|c|c|c|c|}
\hline & \multicolumn{6}{|c|}{ FÁRMACOS ANTIRREUMÁTICOS } \\
\hline & \multicolumn{3}{|c|}{ SEXO } & \multicolumn{3}{|c|}{ EDAD } \\
\hline & Total & Hombres & Mujeres & $14 / 15^{A}$ & $16^{B}$ & $17 / 18^{c}$ \\
\hline Edad media de inicio & $\begin{array}{c}\mathbf{1 4 , 1 9} \\
(d t=1.94) \\
n=52\end{array}$ & $\begin{array}{c}\mathbf{1 4 , 5 8} \\
(\mathrm{dt}=1.74) \\
n=29\end{array}$ & $\begin{array}{c}13,69 \\
(d t=2.10) \\
n=23\end{array}$ & $\begin{array}{c}\mathbf{1 2 , 7 5} \\
(d t=1.39) \\
n=16\end{array}$ & $\begin{array}{c}\mathbf{1 4 , 8 1}^{\mathbf{A}} \\
(\mathrm{dt}=0.98) \\
n=11\end{array}$ & $\begin{array}{c}\mathbf{1 4 , 8 4}^{\mathrm{A}} \\
(\mathrm{dt}=2.09) \\
\mathrm{n}=25\end{array}$ \\
\hline $\begin{array}{l}\text { Continuidad consumo } \\
\text { (Consumo reciente/consumo } \\
\text { alguna vez) }{ }^{*} 100 \\
\text { (Consumo frecuente/consumo } \\
\text { reciente) })^{*} 100\end{array}$ & $\begin{array}{r}61,53 \\
100,00\end{array}$ & $\begin{array}{l}54,83 \\
88,23\end{array}$ & $\begin{array}{r}53,57 \\
100,00\end{array}$ & $\begin{array}{l}50,00 \\
100,00\end{array}$ & $\begin{array}{l}69,23 \\
77,77\end{array}$ & $\begin{array}{r}50,00 \\
100,00\end{array}$ \\
\hline
\end{tabular}

Por el contrario, la edad sí muestra un efecto significativo $[F(2,49)=8.18, p=0.001]$, estableciendo diferencias entre los grupos de 16 a 18 años frente al de menor edad, en el que la edad de inicio en el consumo es aproximadamente tres años inferior.

Analizando la continuidad en el consumo, es en el paso del consumo frecuente al realizado en los últimos treinta días, en el que se produce el mayor aumento de los consumidores ya que el $100 \%$ de los jóvenes que consumieron en los últimos seis meses $(61,5 \%)$ lo continúan haciendo en el último mes, sin que se establezcan diferencias significativas en la proporción de consumidores en función del sexo [88.23 vs. $100.00, Z=1.37$, n.s.] o la edad de los mismos.

En el caso del consumo de fármacos estimulantes (Tabla 7), la edad de inicio en el consumo se sitúa en los 14.7 años, encontrando nuevamente diferencias entre los distintos grupos de edad (para el grupo de $17 / 18$ años es significativamente superior a la de los grupos de 14/15 y 16 años).

Además, tal y como ocurría en el caso del consumo de fármacos antirreumáticos, el $100 \%$ de los consumidores en los últimos seis meses pasa a consumirlos durante los últimos treinta días. También en este caso, el patrón de continuidad en el consumo resultante no presenta en ninguno de los pasos diferencias significativas en función del sexo o la edad de los encuestados.

Para el consumo de derivados morfínicos (Tabla 8), la edad media de inicio en el consumo se sitúa en los 14.0 años. La edad vuelve a mostrar un efecto significativo $(F(2,95=5.83, p \leq 0.01)$, pero en este caso, sólo 


\begin{tabular}{|c|c|c|c|c|c|c|}
\hline & \multicolumn{6}{|c|}{ FÁRMACOS ESTIMULANTES } \\
\hline & \multicolumn{3}{|c|}{ SEXO } & \multicolumn{3}{|c|}{ EDAD } \\
\hline & Total & Hombres & Mujeres & $14 / 15^{A}$ & $16^{\mathrm{B}}$ & $17 / 18^{c}$ \\
\hline Edad media de inicio & $\begin{array}{c}\mathbf{1 4 , 7 6} \\
(\mathrm{dt}=1.53) \\
n=115\end{array}$ & $\begin{array}{c}\mathbf{1 5 , 1 7} \\
(\mathrm{dt}=1.62) \\
\mathrm{n}=34\end{array}$ & $\begin{array}{c}\mathbf{1 4 , 5 9} \\
(\mathrm{dt}=1.47) \\
\mathrm{n}=81\end{array}$ & $\begin{array}{c}\mathbf{1 3}, \mathbf{9 5} \\
(\mathrm{dt}=1.19) \\
n=42\end{array}$ & $\begin{array}{c}\mathbf{1 4 , 7 5 ^ { \mathrm { A } }} \\
(\mathrm{dt}=1.48) \\
\mathrm{n}=41\end{array}$ & 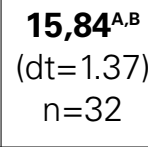 \\
\hline $\begin{array}{l}\text { Continuidad consumo } \\
\text { (Consumo reciente/consumo } \\
\text { alguna vez)*100 } \\
\text { (Consumo frecuente/consumo } \\
\text { reciente) })^{*} 100\end{array}$ & $\begin{array}{c}62,69 \\
100,00\end{array}$ & $\begin{array}{l}67,50 \\
96,29\end{array}$ & $\begin{array}{c}60,46 \\
100,00\end{array}$ & $\begin{array}{c}62,22 \\
100,00\end{array}$ & $\begin{array}{l}56,81 \\
96,00\end{array}$ & $\begin{array}{l}70,27 \\
96,15\end{array}$ \\
\hline
\end{tabular}

\begin{tabular}{|c|c|c|c|c|c|c|}
\hline \multicolumn{7}{|c|}{$\begin{array}{l}\text { Tabla 8. Edad media de inicio y continuidad en el consumo de derivados morfínicos } \\
\text { según sexo y edad }\end{array}$} \\
\hline & \multicolumn{6}{|c|}{ DERIVADOS MORFÍNICOS } \\
\hline & \multicolumn{3}{|c|}{ SEXO } & \multicolumn{3}{|c|}{ EDAD } \\
\hline & Total & Hombres & Mujeres & $14 / 15^{A}$ & $16^{B}$ & $17 / 18^{c}$ \\
\hline Edad media de inicio & $\begin{array}{c}\mathbf{1 4 , 0 4} \\
(\mathrm{dt}=2.07) \\
\mathrm{n}=98\end{array}$ & $\begin{array}{c}\mathbf{1 3 , 6 1} \\
(\mathrm{dt}=2.26) \\
\mathrm{n}=36\end{array}$ & $\begin{array}{c}\mathbf{1 4 , 3 2} \\
(\mathrm{dt}=1.92) \\
\mathrm{n}=61\end{array}$ & $\begin{array}{c}\mathbf{1 3}, \mathbf{2 6} \\
(\mathrm{dt}=1.38) \\
\mathrm{n}=34\end{array}$ & $\begin{array}{c}\mathbf{1 3 , 8 8} \\
(\mathrm{dt}=2.21) \\
\mathrm{n}=26\end{array}$ & $\begin{array}{c}\mathbf{1 4}^{\mathbf{8}} \mathbf{8 4 ^ { \mathrm { A } }} \\
(\mathrm{dt}=2.24) \\
\mathrm{n}=38\end{array}$ \\
\hline $\begin{array}{l}\text { Continuidad consumo } \\
\text { (Consumo reciente/consumo } \\
\text { alguna vez) }{ }^{*} 100 \\
\text { (Consumo frecuente/consumo }_{\text {reciente) }{ }^{*} 100}\end{array}$ & $\begin{array}{l}57,73 \\
86,59\end{array}$ & $\begin{array}{l}44,77^{*} \\
100,00^{*}\end{array}$ & $\begin{array}{l}66,33^{*} \\
80,59^{*}\end{array}$ & $\begin{array}{l}54,38 \\
90,32\end{array}$ & $\begin{array}{l}52,94 \\
85,18\end{array}$ & $\begin{array}{l}65,57 \\
85,00\end{array}$ \\
\hline
\end{tabular}

se marcan diferencias entre el grupo de mayor edad y el de 14/15 años, en el que la edad de comienzo en el consumo es practicamente dos años menor.

En este caso, centrándonos en el consumo de los últimos treinta días, el $86.5 \%$ de los consumidores en el último semestre pasa a un consumo reciente y es en el grupo de los hombres en el que se produce un incremento significativamente superior (100.00 vs. 80.59, $Z=2.59, p \leq 0.05$ ).

En el consumo de tranquilizantes (Tabla 9), la edad media de inicio se sitúa en el mismo rango que en el caso de los estimulantes a los 14.7 años y, como ocurre en el resto de las sustancias, la edad muestra nuevamente un efecto significativo $[F(2,132)=31.02, p=$
0.001 ], estableciéndose diferencias entre los grupos de 16 y 18 años y el de menor edad, en el que la edad de comienzo en el uso de tranquilizantes es de uno (con respecto al grupo de 16 años) a dos años inferior (si se compara con el grupo de 17/18 años).

En los últimos treinta días, el porcentaje de jóvenes que continúan con el consumo se duplica, suponiendo el $89.0 \%$ de los que consumieron en los seis meses anteriores. Además, los datos señalan que es el rango de los 16 años donde aumenta significativamente el porcentaje de jóvenes que abandonan el consumo a pesar de que en edades posteriores vuelva a observarse un notable aumento de la continuidad (79.31 vs. 93.10, $Z=-2.27, p \leq 0.05$ ). 
Tabla 9. Edad media de inicio y continuidad en el consumo de fármacos tranquilizantes según sexo y edad

\begin{tabular}{|c|c|c|c|c|c|c|}
\hline & \multicolumn{6}{|c|}{ FÁRMACOS TRANQUILIZANTES } \\
\hline & \multicolumn{3}{|c|}{ SEXO } & \multicolumn{3}{|c|}{ EDAD } \\
\hline & Total & Hombres & Mujeres & $14 / 15^{A}$ & $16^{\mathrm{B}}$ & $17 / 18^{c}$ \\
\hline Edad media de inicio & $\begin{array}{c}\mathbf{1 4 , 7 6} \\
(\mathrm{dt}=1.80) \\
\mathrm{n}=135\end{array}$ & $\begin{array}{c}\mathbf{1 4 , 5 4} \\
(\mathrm{dt}=2.04) \\
\mathrm{n}=42\end{array}$ & $\begin{array}{c}\mathbf{1 4 , 8 6} \\
(\mathrm{dt}=1.68) \\
\mathrm{n}=93\end{array}$ & $\begin{array}{c}\mathbf{1 3 , 4 6} \\
(d t=1.34) \\
n=45\end{array}$ & $\begin{array}{c}\mathbf{1 4 , 8 2}^{\mathrm{A}} \\
(\mathrm{dt}=1.46) \\
\mathrm{n}=41\end{array}$ & 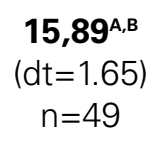 \\
\hline $\begin{array}{l}\text { Continuidad consumo } \\
\text { (Consumo reciente/consumo } \\
\text { alguna vez)* } 100 \\
\text { (Consumo frecuente/consumo } \\
\text { reciente) })^{*} 100\end{array}$ & $\begin{array}{l}48,80 \\
89,02\end{array}$ & $\begin{array}{l}38,18 \\
80,95\end{array}$ & $\begin{array}{l}53,98 \\
91,80\end{array}$ & $\begin{array}{l}42,85 \\
95,83\end{array}$ & $\begin{array}{l}51,78 \\
79,31\end{array}$ & $\begin{array}{r}51,78 \\
93,10^{\mathrm{B}}\end{array}$ \\
\hline
\end{tabular}

Para la cocaína (Tabla 10), la edad media de inicio en el consumo es superior a la del resto de las sustancias analizadas situándose en los 15.5 años, siendo las mujeres la que comienzan a consumir cocaína a una edad más temprana (prácticamente la iniciación en el uso es un año anterior).

Tabla 10. Edad media de inicio y continuidad en el consumo de cocaína según sexo y edad

\begin{tabular}{|c|c|c|c|c|c|c|}
\hline & \multicolumn{6}{|c|}{ COCAÍNA } \\
\hline & \multicolumn{3}{|c|}{ SEXO } & \multicolumn{3}{|c|}{ EDAD } \\
\hline & Total & Hombres & Mujeres & $14 / 15^{A}$ & $16^{\mathrm{B}}$ & $17 / 18^{c}$ \\
\hline Edad media de inicio & $\begin{array}{c}\mathbf{1 5 , 5 9} \\
(d t=1.41) \\
n=42\end{array}$ & $\begin{array}{c}\mathbf{1 5}, \mathbf{9 2 *} \\
(\mathrm{dt}=1.44) \\
\mathrm{n}=26\end{array}$ & $\begin{array}{c}\mathbf{1 5 , 0 6 *} \\
(\mathrm{d} t=1.24) \\
n=16\end{array}$ & $\begin{array}{c}13,57 \\
(d t=0.53) \\
n=7\end{array}$ & $\begin{array}{c}\mathbf{1 5 , 1 6} \\
(d t=1.11) \\
n=12\end{array}$ & $\begin{array}{c}\mathbf{1 6 , 4 3 ^ { \mathrm { A } , \mathrm { B } }} \\
(\mathrm{dt}=0.94) \\
\mathrm{n}=23\end{array}$ \\
\hline $\begin{array}{l}\text { Continuidad consumo } \\
\text { (Consumo reciente/consumo } \\
\text { alguna vez) }{ }^{*} 100 \\
\text { (Consumo frecuente/consumo } \\
\text { reciente)* } 100\end{array}$ & $\begin{array}{l}54,90 \\
89,28\end{array}$ & $\begin{array}{l}56,66 \\
94,11\end{array}$ & $\begin{array}{l}52,38 \\
81,81\end{array}$ & $\begin{array}{r}62,50 \\
100,00\end{array}$ & $\begin{array}{l}42,85 \\
83,33\end{array}$ & $\begin{array}{l}56,66 \\
82,35\end{array}$ \\
\hline
\end{tabular}

Atendiendo a la continuidad en el consumo, el $54.9 \%$ de los que probaron en alguna ocasión esta sustancia lo ha continuado haciendo durante los seis últimos meses y, de ellos, el $89.2 \%$ sigue consumiendo en el último mes.

Finalmente, en el caso del consumo de drogas de síntesis (Tabla 11), la edad de inicio en el mismo es superior a la del resto de drogas de comercio ilegal (15.5 años) y la edad vuelve a mostrar efectos determinantes $[F(2,103)=31.45, p=0.001]$, siendo la edad de inicio en el grupo de 17/18 años significativamente superior a la de los restantes grupos.
En el consumo en los últimos treinta días, la proporción de jóvenes que continúan usando drogas en este período vuelve a incrementarse suponiendo el $86.4 \%$ de los que las consumían en los seis meses anteriores. En este caso, sí se observan diferencias entre los grupos de edad puesto que la proporción de consumidores que continúan utilizando este tipo de sustancias es significativamente superior en el grupo de 14 y 15 años frente al de 16 años (94.44 vs. 64.28, $Z=2.34, p \leq 0.05$ ) y en el de jóvenes de 17 y 18 años frente a este último grupo de edad (64.28 vs. 97.29, $Z=-3.51, p \leq 0.05)$ en el que se observa un mayor porcentaje de abandonos en el consumo. 


\begin{tabular}{|c|c|c|c|c|c|c|}
\hline \multicolumn{7}{|c|}{$\begin{array}{l}\text { Tabla 11. Edad media de inicio y continuidad en el consumo de drogas sintéticas } \\
\text { según sexo y edad }\end{array}$} \\
\hline & \multicolumn{6}{|c|}{ DROGAS SINTÉTICAS } \\
\hline & \multicolumn{3}{|c|}{ SEXO } & \multicolumn{3}{|c|}{ EDAD } \\
\hline & Total & Hombres & Mujeres & $14 / 15^{A}$ & $16^{\mathrm{B}}$ & $17 / 18^{c}$ \\
\hline Edad media de inicio & $\begin{array}{c}\mathbf{1 5 , 5 2} \\
(\mathrm{dt}=1.30) \\
\mathrm{n}=106\end{array}$ & $\begin{array}{c}\mathbf{1 5 , 6 6} \\
(\mathrm{dt}=1.32) \\
\mathrm{n}=63\end{array}$ & $\begin{array}{c}\mathbf{1 5 , 2 6} \\
(\mathrm{dt}=1.26) \\
\mathrm{n}=41\end{array}$ & $\begin{array}{c}\mathbf{1 4 , 1 2} \\
(\mathrm{d} t=1.36) \\
\mathrm{n}=24\end{array}$ & 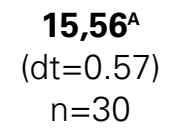 & $\begin{array}{c}\mathbf{1 6 , 1 5}^{\mathrm{A}, \mathrm{B}} \\
(\mathrm{dt}=1.07) \\
n=52\end{array}$ \\
\hline $\begin{array}{l}\text { Continuidad consumo } \\
\text { (Consumo reciente/consumo } \\
\text { alguna vez) }{ }^{*} 100 \\
\text { (Consumo frecuente/consumo } \\
\text { reciente) }{ }^{*} 100\end{array}$ & $\begin{array}{l}66,94 \\
86,41\end{array}$ & $\begin{array}{l}65,33 \\
89,79\end{array}$ & $\begin{array}{l}69,56 \\
81,25\end{array}$ & $\begin{array}{l}78,26 \\
94,44\end{array}$ & $\begin{array}{l}70,00 \\
64,28^{A}\end{array}$ & $\begin{array}{l}61,66 \\
97,29^{8}\end{array}$ \\
\hline
\end{tabular}

\section{DISCUSIÓN}

Los datos obtenidos muestran cómo el cannabis es la sustancia más consumida por los jóvenes ofreciendo una prevalencia de consumo muy superior a la obtenida para el resto de las drogas estimadas (practicamente el $19 \%$ de los encuestados afirman haberla consumido en el mes previo a la encuesta), confirmándose como la de mayor penetración en la población más joven (PNSD, 1994,1996; Elzo, 1996; Torres y Domínguez, 1997). Esta situación se hace más evidente cuando se observa que esta sustancia junto con los estimulantes son las que los jóvenes consumen en mayor medida con una frecuencia semanal, llegando a porcentajes cercanos al $65 \%$ de los consumidores en el último mes. Con respecto al resto de las drogas consideradas, en nuestra muestra, son los derivados morfínicos, los estimulantes, los tranquilizantes y las drogas de síntesis las que alcanzan mayores prevalencias de consumo después del hachís, siendo la cocaína y los fármacos antirreumáticos los menos utilizados por los jóvenes encuestados.

En este apartado, para la lectura de las conclusiones obtenidas en el presente trabajo y su posible generalización y comparación con otros estudios, es necesario recordar la tipología de las drogas de comercio ilegal que se han estimado en el mismo. En primer lugar, al igual que otros autores, se analiza el consumo de sustancias consideradas de forma independiente como el cannabis, la cocaína y las drogas de síntesis. En segundo lugar, se analizan distintos grupos de sustancias de la misma familia que suponen la inclusión de otras que no han sido consideradas por otros trabajos de la misma área, como es el caso de los derivados morfínicos además de los fár- macos estimulantes y los tranquilizantes en los que, como en otros estudios, se consideraron todo tipo de psicofármacos de uso común que provocaran efectos similares. En tercer lugar, se ha incluido el estudio del consumo de fármacos antirreumáticos que, por sugerencia de los tutores de los centros, podían también ser de los más utilizados por la población de jóvenes adolescentes al ser indicados para el tratamiento de dolencias típicas de esta edad provocados por el deporte o la actividad física. Así pues, en cuanto a la frecuencia de consumo de las diferentes sustancias, los resultados obtenidos son notablemente distintos a los de otros trabajos. En este sentido, sorprende que, por ejemplo, los derivados morfínicos ocupen el segundo lugar después del cannabis o que los medicamentos antirreumáticos sean más consumidos que la cocaína.

De cualquier forma, este tipo de resultados bien puede señalar la existencia de un conjunto de sustancias de uso común entre la población más joven que necesariamente deben ser consideradas al tratarse de drogas, que aunque médicas, tienen un poder adictivo similar a las consideradas tradicionalmente $y$, lo que es más alarmante, que pueden ser de fácil acceso a estas edades por encontrarse entre las prescritas más comúnmente para sí mismos o para sus familiares adultos. Asimismo, esta propuesta explicativa de los altos índices de consumo centrada en la mayor accesibilidad del conjunto de drogas médicas por parte de los adolescentes, podría verse apoyada por el hecho de que la edad media de inicio para el total de estas sustancias se encuentre en torno a los 14 años mientras que, para otras como la cocaína, el cannabis y las drogas de síntesis es un año y medio posterior, al ser posiblemente éstas las que requieren una 
mayor planificación para su obtención y, como no, un mayor poder adquisitivo.

Por el contrario, en el caso de la cocaína y con respecto a la prevalencia del consumo llevado a cabo durante el mes previo a la encuesta, sí se obtienen resultados muy parecidos a los constatados por otros estudios como el realizado a nivel nacional por el PNSD (1996) o a nivel comarcal como el llevado a cabo recientemente en la Comunidad gallega (EDIS, 1996) y en la andaluza (Junta de Andalucía, 1997), y no así en el de los estimulantes (PNSD, 1994; Lora et al., 1996; Junta de Andalucía, 1997) y la drogas de síntesis (PNSD, 1994; EDIS, 1996; Junta de Andalucía, 1997) en el que la proporción de jóvenes consumidores es considerablemente superior.

Como era de esperar por estudios previos, en el caso de los tranquilizantes se han obtenido prevalencias de consumo menores que en otras zonas como Andalucía (Lora et al., 1996; Junta de Andalucía, 1997) y Galicia (Martínez, García, Domingo y Machín, 1996) pero notablemente superiores a las obtenidas a nivel nacional (PNSD, 1994).

Para el grupo de drogas de comercio ilegal y a diferencia de lo que ocurría con el tabaco y el alcohol, sí aparecen diferencias señaladas en el porcentaje de consumidores de cada una de las sustancias en función del sexo de los mismos. Así pues, por una parte, son los hombres los que consumen en mayor medida el cannabis confirmando los resultados obtenidos por diversos estudios que asocian repetidamente el consumo de esta sustancia al sexo masculino (PNSD, 1994,1996; Campins et al., 1996; CAM, 1996,1997; Junta de Andalucía, 1997; Torres y Domínguez, 1997) y, por otra, es el porcentaje de mujeres consumidoras de tranquilizantes (PNSD, 1994,1996; Junta de Andalucía, 1997), derivados morfínicos y fármacos estimulantes el que supera al de los hombres, volviendo a señalar el hecho constatado de la preferencia por parte del sexo femenino por el uso de fármacos, que posiblemente suponga un consumo menos exteriorizado, más centrado en sustancias de mayor accesibilidad y con menos connotaciones a nivel social (supuestamente, se trata de fármacos que a pesar de exigir receta médica para su comercialización pueden tener una mayor aceptación social al ser indicadas, realizando un buen uso de las mismas, para el tratamiento de dolencias o problemas relacionados con la salud).

Además de estas diferencias encontradas en la proporción de jóvenes consumidores según su sexo, también el patrón de continuidad en el uso de cada una de las sustancias de comercio ilegal difiere en relación con esta variable y dependiendo de la droga de referencia. Así pues, el porcentaje de hombres que mantienen el uso de cannabis, derivados morfínicos, cocaína y drogas de síntesis es mayor que el de las mujeres y, por el contrario, son éstas las que reafir- man su consumo en el caso de los fármacos antirreumáticos, los estimulantes y los tranquilizantes (cifras superiores a las obtenidas por el PNSD en 1994).

Finalmente, subrayar que nuevamente el consumo de este tipo de drogas mantiene una relación directa con la edad, siendo el grupo de jóvenes de entre los 16 y los 18 años el de mayor riesgo puesto que, a estas edades, la proporción de consumidores incrementa notablemente con respecto a los grupos de menor edad (PNSD, 1994; CAM, 1996, 1997).

\section{BIBLIOGRAFÍA}

CAMPINS, M., GASCH, J., HEREU, P., ROSSELLÓ, J. Y VAQUÉ, J. (1996). Consumo y actitudes de los adolescentes frente a sustancias adictivas: Encuesta de prevalencia. Anales de Pediatría, 45(5), 475-478.

COMAS, D. (1994). Los jóvenes y el uso de drogas en la España de los años 90. Madrid: Ministerio de Asuntos Sociales. Instituto de la Juventud.

CONSEJERÍA DE SANIDADY SERVICIOS SOCIALES DE LA COMUNIDAD DE MADRID (1996). Informe sobre los hábitos de salud en la población juvenil de la Comunidad de Madrid. Boletín Epidemiológico de la Comunidad de Madrid, 4, 3-15.

CONSEJERÍA DE SANIDADY SERVICIOS SOCIALES DE LA COMUNIDAD DE MADRID (1997). Informe sobre los hábitos de salud en la población juvenil de la Comunidad de Madrid. Boletín Epidemiológico de la Comunidad de Madrid, 5, 3-17.

EDIS (1996). El consumo de drogas en Galicia IV. Santiago: Plan Autonómico de Drogodependencias.

ELZO, J. (1996). Drogas y Escuela V. Bilbao: Departamento de Justicia, Economía, Trabajo y Seguridad Social. Secretaría de Drogodependencias.

JUNTA DE ANDALUCÍA (1997). Plan andaluz sobre drogas. Sevilla: Consejería de Salud. Comisionado para la droga.

LORA, N., RUÍZ, R., JIMÉNEZ, J.M., PERULA DE TORRES, L.A., VICENTERUEDA, J. Y RODRÍGUEZ, F. (1996). Prevalencia del consumo de drogas entre los escolares de B.U.P. de Córdoba. Adicciones, 8(2), 219-234.

MARTÍNEZ, J., GARCÍA, J., DOMINGO, M. Y MACHÍN, A.J. (1996). Consumo de alcohol, tabaco y drogas en adolescentes. Atención Primaria, 18, 383-385.

MENDOZA, R., SAGRERA, M.R. Y BATISTA, J.M. (1992). Conductas de los escolares españoles relacionadas con la salud. Madrid: Ministerio de Sanidad y Consumo.

PLAN NACIONAL SOBRE DROGAS (1994). Encuesta sobre drogas a la población escolar. Madrid: Delegación del Gobierno para el Plan Nacional sobre Drogas.

PLAN NACIONAL SOBRE DROGAS (1996). Encuesta sobre drogas a la población escolar. Madrid: Delegación del Gobierno para el Plan Nacional sobre Drogas. 
PLAN NACIONAL SOBRE DROGAS: Memoria 1995. Madrid: Ministerio del Interior.

TORRES, A.J. Y DOMínGUEZ, M.D. (1997). Consumo de drogas ilegales y variables sociodemográficas en la adolescencia: Estudio epidemiológico comunitario en Galicia. Revista de Psiquiatría Infanto Juvenil, 1, 4-9. 